\title{
A UNIQUE CONTINUATION THEOREM INVOLVING A DEGENERATE PARABOLIC OPERATOR
}

\author{
ALAN V. LAIR
}

Abstract. We consider the degenerate parabolic operator $L[u]=\gamma B[u]-$ $u_{t}$ on a domain $D=\Omega \times(0, T]$ where $B[u]=\sum_{i, j=1}^{n}\left(a_{i j}(x) u_{x_{j}}\right)_{x_{i}}$ and $\gamma$ is an arbitrary complex number. Classically, $\gamma=1$ and the real-valued matrix $\left(a_{i j}\right)$ is positive definite. We assume $\left(a_{i j}\right)$ is a real-valued symmetric matrix but not necessarily definite. We prove that any complex-valued function $u$ which satisfies the inequality $|L[u]|<c|u|$ for some nonnegative constant $c$ and vanishes initially as well as on the boundary of $\Omega$ must vanish on all of $D$. The theorem is particularly useful in studying uniqueness for many systems which are not parabolic.

1. Introduction. We consider the differential operator

$$
L \equiv \gamma B-\partial / \partial t,
$$

where $\gamma$ is a complex number, and

$$
B[u] \equiv \sum_{i, j=1}^{n}\left(a_{i j}(x) u_{x_{j}}\right)_{x_{i}}
$$

in which the real-valued matrix $\left(a_{i j}(x)\right)$ defined on a domain $\Omega$ is assumed to be symmetric but not necessarily definite. We are using subscripts to denote differentiation.

A unique continuation theorem is established for the differential inequality

$$
|L[u]|^{2} \leqslant c|u|^{2}
$$

where $c$ is a nonnegative constant. Such results are useful in the study of uniqueness for systems of equations of the form (See [1].)

$$
u_{t}^{m}=\sum_{l=1}^{N} \sum_{i, j=1}^{n}\left(a_{i j}(x) e^{l m} u_{x_{j}}^{l}\right)_{x_{i}}+f_{m}(x, t, u)
$$

for $1 \leqslant m \leqslant N$. In the event that the constant matrix $\left(e^{l m}\right)$ has complex eigenvalues, classical results are not applicable to the system (1.4).

The problem of unique continuation for linear parabolic equations was considered by Lees and Protter [4] who generalized the results of several authors by considering differential inequalities of the form

$$
(L[v])^{2} \leqslant c_{1} t^{-2} v^{2}+c_{2} \sum_{m=1}^{n}\left(v_{x_{m}}\right)^{2}
$$

Received by the editors October 11, 1976.

AMS (MOS) subject classifications (1970). Primary 35B45, 35K 10.

Key words and phrases. Degenerate parabolic operator, unique continuation.

c American Mathematical Society 1977 
in which it was assumed that $\gamma=1$ in (1.1) and that the matrix $\left(a_{i j}\right)$ be time dependent and satisfy the usual parabolicity condition of being positive definite.

We consider complex-valued functions $u\left(x_{1}, \ldots, x_{n}, t\right)$ which satisfy (1.3) and which vanish initially as well as on the boundary of $\Omega$. The proofs require considerably sharper integral estimates than those used by Lees and Protter [4] although the methods of the proofs are essentially the same.

2. The main results. Let $D=\Omega \times(0, T]$ where $\Omega$ is a bounded open connected set in real $n$-dimensional Euclidean space, $R^{n}$, with a sufficiently smooth boundary $(\partial \Omega)$ to ensure the validity of integration by parts.

We shall say that the differential operator $L$ defined in (1.1) and (1.2) satisfies condition $(\mathrm{G})$ if the real-valued symmetric matrix $\left(a_{i j}\right)$ is continuous on $\bar{\Omega}$ with continuous bounded first order derivatives on $\Omega$. The usual parabolicity condition that the matrix $\left(a_{i j}\right)$ be (positive) definite is not required here. We shall use the notation

$$
\begin{aligned}
(v, w) & =\int_{D} v(x, t) \bar{w}(x, t) d x d t, \\
\|v\| & =(v, v)^{1 / 2} .
\end{aligned}
$$

We let $\tilde{P}$ be the set of complex-valued functions $v$ such that $v$ is continuous on $\bar{D}$, the second partial derivatives of $v$ in $x$ are continuous and square integrable on $D$, and the first partial derivatives of $v_{t}$ in $x$ are continuous and square integrable on $D$. We let $P$ be the set of funtions in $\tilde{P}$ which vanish on the boundary of $D$. Finally we let $\lambda(t)=t+\eta$ where $\eta$ is any fixed positive number.

Before proving the main result of the paper, we need two preliminary lemmas.

LEMMA 1. If the operator $L$ satisfies condition (G), $u \in P, k$ is any positive integer and $z(x, t)=[\lambda(t)]^{-k} u(x, t)$, then

$$
\|\gamma B[z]\|^{2}+\left\|z_{t}\right\|^{2}+k^{2}\left\|\lambda^{-1} z\right\|^{2} \geqslant 2 \operatorname{Re}\left(\gamma B[z], z_{t}+k \lambda^{-1} z\right) .
$$

Proof. We let $v=\operatorname{Re}(z), w=\operatorname{Im}(z), \alpha=\operatorname{Re}(\gamma)$ and $\beta=\operatorname{Im}(\gamma)$. We now obtain

$$
\begin{aligned}
\operatorname{Re}\left(\gamma B[z], z_{t}\right)= & \alpha\left(B[v], v_{t}\right)+\alpha\left(B[w], w_{t}\right) \\
& +\beta\left(B[v], w_{t}\right)-\beta\left(B[w], v_{t}\right) .
\end{aligned}
$$

However, in view of the symmetry of $\left(a_{i j}\right)$, we have

$$
\begin{aligned}
\left(B[v], v_{t}\right) & =-\sum_{i, j=1}^{n}\left(a_{i j} v_{x_{i}}, v_{x_{j}}\right) \\
& =-\sum_{i, j=1}^{n} \int_{D}\left[a_{i j} / 2\right]\left[v_{x_{i}} v_{x_{j}}\right]_{t} d x d t=0 .
\end{aligned}
$$

Similarly, we get 


$$
\left(B[w], w_{t}\right)=0 .
$$

Equation (2.4) combined with (2.5) and (2.6) yields

$$
\operatorname{Re}\left(\gamma B[z], z_{t}\right)=\beta\left(B[v], w_{t}\right)-\beta\left(B[w], v_{t}\right) .
$$

We also have

$$
\begin{aligned}
\operatorname{Re}\left(\gamma B[z], k \lambda^{-1} z\right)= & \alpha\left(B[v], k \lambda^{-1} v\right)+\alpha\left(B[w], k \lambda^{-1} w\right) \\
& +\beta\left(B[v], k \lambda^{-1} w\right)-\beta\left(B[w], k \lambda^{-1} v\right) .
\end{aligned}
$$

Since $B$ is a symmetric operator, the last two terms in (2.8) add to zero. Thus (2.8) becomes

$$
\operatorname{Re}\left(\gamma B[z], k \lambda^{-1} z\right)=\alpha\left(B[v], k \lambda^{-1} v\right)+\alpha\left(B[w], k \lambda^{-1} w\right) .
$$

With the use of (2.7) and (2.9), we get

$$
\begin{aligned}
\|\dot{\gamma} B[z]\|^{2}+ & \left\|z_{t}\right\|^{2}+\left\|k \lambda^{-1} z\right\|^{2}-2 \operatorname{Re}\left(\gamma B[z], z_{t}+k \lambda^{-1} z\right) \\
= & \left(\alpha^{2}+\beta^{2}\right)\|B[z]\|^{2}+\left\|z_{t}\right\|^{2}+\left\|k \lambda^{-1} z\right\|^{2} \\
& -2 \beta\left(B[v], w_{t}\right)+2 \beta\left(B[w], v_{t}\right)-2 \alpha\left(B[v], k \lambda^{-1} v\right) \\
& -2 \alpha\left(B[w], k \lambda^{-1} w\right) \\
= & \alpha^{2}\|B[v]\|^{2}-2 \alpha\left(B[v], k \lambda^{-1} v\right)+\left\|k \lambda^{-1} v\right\|^{2} \\
& +\beta^{2}\|B[v]\|^{2}-2 \beta\left(B[v], w_{t}\right)+\left\|w_{t}\right\|^{2} \\
& +\alpha^{2}\|B[w]\|^{2}-2 \alpha\left(B[w], k \lambda^{-1} w\right)+\left\|k \lambda^{-1} w\right\|^{2} \\
& +\beta^{2}\|B[w]\|^{2}+2 \beta\left(B[w], v_{t}\right)+\left\|v_{t}\right\|^{2} \\
= & \left\|\alpha B[v]-k \lambda^{-1} v\right\|^{2}+\left\|\beta B[v]-w_{t}\right\|^{2} \\
& +\left\|\alpha B[w]-k \lambda^{-1} w\right\|^{2}+\left\|\beta B[w]+v_{t}\right\|^{2} .
\end{aligned}
$$

Since the last term in (2.10) is nonnegative, this completes the proof.

LEMmA 2. If the operator $L$ satisfies condition (G), $u \in P$, and $k$ is any positive integer, then

$$
\left\|\lambda^{-k} L[u]\right\|^{2} \geqslant k\left\|\lambda^{-k-1} u\right\|^{2}
$$

Proof. Let $z(x, t)=[\lambda(t)]^{-k} u(x, t)$. Then $z \in P$ and $L[u]=L\left[\lambda^{k} z\right]=$ $\gamma \lambda^{k} B[z]-\lambda^{k} z_{t}-k \lambda^{k-1} z$. This identity yields

$$
\begin{aligned}
\left\|\lambda^{-k} L[u]\right\|^{2}= & \left\|\gamma B[z]-z_{t}-k \lambda^{-1} z\right\|^{2} \\
= & \|\gamma B[z]\|^{2}+\left\|z_{t}\right\|^{2}+k^{2}\left\|\lambda^{-1} z\right\|^{2} \\
& -2 \operatorname{Re}\left(\gamma B[z], z_{t}+k \lambda^{-1} z\right)+2 \operatorname{Re}\left(k \lambda^{-1} z, z_{t}\right) .
\end{aligned}
$$

However, the last term in (2.12) may be written as 


$$
\begin{aligned}
2 \operatorname{Re}\left(k \lambda^{-1} z, z_{t}\right) & =k \int_{D} \lambda^{-1}\left[|z|^{2}\right]_{t} d x d t=k \int \lambda^{-2}|z|^{2} d x d t \\
& =k\left\|\lambda^{-1} z\right\|^{2}=k\left\|\lambda^{-k-1} u\right\|^{2} .
\end{aligned}
$$

Combining (2.12) and (2.13), we have

$$
\begin{aligned}
& \left\|\lambda^{-k} L[u]\right\|^{2}-k\left\|\lambda^{-k-1} u\right\|^{2} \\
& \quad=\|\gamma B[z]\|^{2}+\left\|z_{t}\right\|^{2}+\left\|k \lambda^{-1} z\right\|^{2}-2 \operatorname{Re}\left(\gamma B[z], z_{t}+k \lambda^{-1} z\right) .
\end{aligned}
$$

From Lemma 1, we know that the right-hand side of equation (2.14) is nonnegative and this completes the proof.

THEOREM. Suppose $u \in \tilde{P}$, the operator $L$ satisfies condition (G), and

$$
\begin{aligned}
|L[u]|^{2} & \leqslant c|u|^{2}, \quad(x, t) \in D, \\
u(x, 0) & =0, \quad x \in \Omega, \\
u(s, t) & =0, \quad(s, t) \in \partial \Omega \times[0, T] .
\end{aligned}
$$

Then $u(x, t)=0$ for all $(x, t) \in D$.

Proof. Suppose $t_{1} \in(0, T)$. We wish to show $u(x, t)=0$ for all $t \in\left[0, t_{1}\right]$, $x \in \Omega$. For this purpose choose $0<t_{1}<t_{2}<t_{3}<T$. Let $\zeta$ be a real-valued infinitely differentiable function defined on $[0, T]$ such that $\zeta(t)=1$ for $t \in\left[0, t_{2}\right], \zeta(t)=0$ for $t \in\left[t_{3}, T\right]$, and $0 \leqslant \zeta(t) \leqslant 1$ for $t \in\left[t_{2}, t_{3}\right]$. Now set $v(x, t)=\zeta(t) u(x, t)$ and observe that $v \in P$. With the use of elementary methods and Lemma 2, we have

$$
\begin{aligned}
& \left(t_{2}+\eta\right)^{-2 k} \int_{t_{2}}^{t_{3}} \int_{\Omega}|L[v]|^{2} d x d t \geqslant \int_{t_{2}}^{t_{3}} \int_{\Omega} \lambda^{-2 k}|L[v]|^{2} d x d t \\
& \quad=\left\|\lambda^{-k} L[v]\right\|^{2}-\int_{0}^{t_{2}} \int_{\Omega} \lambda^{-2 k}|L[v]|^{2} d x d t \\
& \geqslant k\left\|\lambda^{-k-1} v\right\|^{2}-c \int_{0}^{t_{2}} \int_{\Omega} \lambda^{-2 k}|v|^{2} d x d t \\
& \geqslant k \int_{0}^{t_{2}} \int_{\Omega} \lambda^{-2 k-2}|v|^{2} d x d t-c \eta^{-2} \int_{0}^{t_{2}} \int_{\Omega} \lambda^{-2 k-2}|v|^{2} d x d t \\
& =\left(k-c \eta^{-2}\right) \int_{0}^{t_{2}} \int_{\Omega} \lambda^{-2 k-2}|v|^{2} d x d t
\end{aligned}
$$

For $k>c \eta^{-2}$, we obtain

$$
\begin{aligned}
& \left(k-c \eta^{-2}\right) \int_{0}^{t_{2}} \int_{\Omega} \lambda^{-2 k-2}|v|^{2} d x d t \\
& \quad \geqslant\left(k-c \eta^{-2}\right)\left(t_{1}+\eta\right)^{-2 k-2} \int_{0}^{t_{1}} \int_{\Omega}|v|^{2} d x d t .
\end{aligned}
$$

Combining (2.16) and (2.17) and simplifying, we get 


$$
\begin{gathered}
{\left[\eta^{2} /\left(\eta^{2} k-c\right)\right]\left[\left(t_{1}+\eta\right) /\left(t_{2}+\eta\right)\right]^{2 k} \int_{t_{2}}^{t_{3}} \int_{\Omega}|L[v]|^{2} d x d t} \\
\geqslant\left(t_{1}+\eta\right)^{-2} \int_{0}^{t_{1}} \int_{\Omega}|v|^{2} d x d t .
\end{gathered}
$$

Since $t_{1}<t_{2}$, the left-hand side of (2.18) approaches zero as $k \rightarrow \infty$ and this implies the right-hand side is zero since it is independent of $k$. This completes the proof.

\section{REFERENCES}

1. J. R. Cannon, W. T. Ford and A. V. Lair, Quasilinear parabolic systems, J. Differential Equations 20 (1976), 441-472. MR 53 \# 1028.

2. A. Friedman, Partial differential equations of parabolic type, Prentice-Hall, Englewood Cliffs, N. J., 1964. MR 31 \#6062.

3. O. A. Ladyženskaja, V. A. Solonnikov and N. N. Ural'ceva, Linear and quasilinear equations of parabolic type, Transl. Math. Monographs, vol. 23, Amer. Math. Soc., Providence, R. I., 1968. MR 39 \#3159a,b.

4. M. Lees and M. H. Protter, Unique continuation for parabolic differential equations and inequalities, Duke Math. J. 28 (1961), 369-382.

5. H. Yamabe, A unique continuation theorem of a diffusion equation, Ann. of Math. 69 (1959), 462-466. MR 21 \#206.

Department of Mathematics, University of South Dakota, Vermillion, South Dakota 57069 